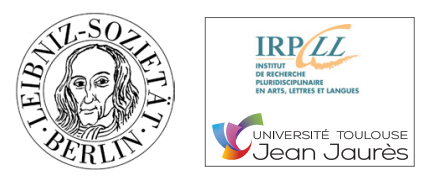

\title{
Études culturelles et études germaniques en France
}

\section{Françoise Lartillot}

Université de Lorraine

\section{Abstract}

L'article retrace à grands traits l'histoire de la rencontre entre études germaniques et Cultural Studies / études culturelles, comportant pour une part une interrogation quant à l'organon des questionnements de ces dernières. Toutefois, la pluridisciplinarité pratiquée de longue date par les germanistes français les conduit à se rapprocher des études culturelles. Certaines évolutions institutionnelles, comme la création du CIERA et du centre Marc Bloch par exemple, des pratiques novatrices apportées par les études culturelles, notamment dans le cadre de cotutelles de thèse ou des programmes des Équipes d'Accueil en sont la preuve. Ces éléments novateurs sont les bienvenus s'inscrivant dans le cadre d'une conception globalement humboldtienne de l'université mais soucieuse également de ne pas réifier le savoir.

In diesem Beitrag wird die Geschichte der Begegnung zwischen der französischen Germanistik und den Cultural Studies / études culturelles in einer weiten Perspektive dargestellt, in deren Kontext nicht zuletzt das Organon der Cultural Studies / études culturelles hinterfragt wird. Gleichwohl führt die Pluridisziplinarität, die seit geraumer Zeit die französische Germanistik prägt, die französischen Germanist innen dazu, an die études culturelles anzuschließen. Institutionnelle Entwicklungen wie die Gründung des CIERA oder des Centre Marc Bloch etwa, neue Forschungsmethoden, die mit den études culturelles verbunden sind, und die im Rahmen von Cotutelles-Verfahren oder von Programmen von Forschungszentren Anwendung finden, belegen dies. Diese innovativen Schritte werden positiv aufgenommen, indem sie sich dem Paradigma der Humboldtschen Universität verpflichten, die ihrerseits das Wissen nicht verdinglichen will.

This article broadly reviews the history of the encounter between French German studies and Cultural Studies / études culturelles. In this context, not least the organon of the latter is called into question. Yet, the long-established multidisciplinary approach of French specialists in German studies has led them to move closer to Cultural Studies. Institutional developments, such as the creation of the CIERA and the Marc Bloch Centre, innovative research practices and methods brought about by cultural studies and applied particularly within the framework of joint PhD programs or research center projects, are proof of this. These innovative steps, committing themselves to the paradigm of Humboldtian university, which for its part refuses to reify knowledge, are received positively.

\section{Keywords}

Etudes germaniques $\bullet$ interdisciplinarité $\bullet$ études culturelles $\bullet$ cultural studies $\bullet$ organon des questionnements $\bullet$ interculturalité

Französische Germanistik • Interdisziplinarität • études culturelles • cultural studies • Organon der Fragestellung • Interkulturalität • French German studies

Interdisciplinarity $\bullet$ études culturelles $\bullet$ cultural studies $\bullet$ organon of questions $\bullet$ interculturality

Essayons d'obérer la diversité des études culturelles en elles-mêmes et leur " coloration » " identitaire » - autrement dit d'obèrer le fait que les études culturelles, les Cultural Studies et les Kulturgeschichtliche Studien sont déjà en elles-mêmes des branches diversifiées d'un phénomène de mutation scientifique "tumultueuse et brouillonne " (Saint-Jacques 2011 : 155 ; Musner 2004 : 61-76 ; ChalardFillaudeau $2015: 6-8$ ) -, et résumons les études culturelles comprises dans leur «typicité idéale ${ }^{1}:$ on obtiendra alors

1 Etant donné que différents articles détaillent ces notions et leur évolution dans le présent numéro, nous ne revenons pas ici sur le fait que, en particulier depuis la fin des années 1960, les études culturelles ont connu une large diffusion dans les universités britanniques puis nord-américaines (Brantlinger 1990/2013). la présentation d'éléments définitionnels communs tels que le croisement disciplinaire (qu'il est convenu de nommer « transdisciplinarité » au sens de la transformation des humanités en études culturelles telle qu'elle est passée dans les usages des universitaires nord-américains) et l'ouverture, le décloisonnement, la remise en cause des hiérarchies implicites. On observera aussi l'association à des champs de recherche tels que, pour citer quelques sous-ensembles des Cultural Studies et sans détailler les interférences complexes qui existent entre approches anglaise, américaine et française (Mattelart / Neveu 2003/2008/2010) : « culture populaire ", " féminisme ", " gender studies » - entre autres dans le sillage de Michel Foucault et des philosophes de la déconstruction qui incitèrent à revisiter les phénomènes culturels 
par le truchement des discours et des représentations de la différence des sexes -, " areal studies " voire " transareal studies " - au sens de circulation des savoirs aussi bien que des catégories littéraires dans une même langue et dans le cadre de la globalisation (Ette 2001) ; on citera évidemment aussi, en particulier à partir de Said et de sa réception à la fin des années 1970, le postcolonialisme mais on peut mentionner un courant plus récent, le posthumain, lequel est issu de la cybernétique, de la science-fiction, de l'inquiétude ou du moins de la conscience des limites aujourd'hui imposées à l'esprit humain par le corps biologique (Michaud 2006).

Enfin, on notera l'association à des orientations méthodologiques telles que " sociologie » ou " sémiotique ». A consulter les passages consacrés à leur méthodologie, les jeunes chercheurs auteurs de mémoires de thèse, s'ils placent leur approche sous l'égide des sciences de la culture, utilisent des dénominations variables. En effet, on trouve leur approche qualifiée tantôt de " croisement disciplinaire ", ce qui implique que l'accent est mis sur deux ou plusieurs ancrages scientifiques, sachant qu'il est fréquent de mettre ainsi en perspective l'analyse du discours esthétisé voire fictionnel et les données factuelles, surtout historiques et sociales. S'il est précisé qu'il s'agit de "pluridisciplinarité » et si l'on entend par là la juxtaposition de spécificités dont le chercheur exploite les convergences, c'est une approche qui les incite à travailler par exemple sur la circulation de savoirs spécialisés et sur les procédés de leur large diffusion (par exemple des conseils médicaux divulgués par le truchement de supports textuels identifiés comme destinés à un public non spécialisé et populaire). "L'interdisciplinarité » est plus souvent à l'œuvre dans ce genre de thèses placées sous l'égide des sciences de la culture car elle consiste à importer des catégories et à osciller d'une discipline à l'autre, ce qui facilite les dialogues, les échanges et les transferts non seulement des concepts mais aussi des méthodes, ce dernier aspect méritant d'être souligné. Plus ambitieuse et prometteuse, même si c'est une dénomination qui se trouve plus rarement dans ces études, la « transdisciplinarité ", qui transcende les frontières des disciplines, contribue à ajuster les savoirs aux attentes culturelles et à la permanente évolution des connaissances et des techniques, et à inventer des modèles qui possèdent des références communes à un ensemble de disciplines. Un intéressant exemple en est la notion d'anthropocène : initialement réservé aux géographes, climatologues, géologues, biologistes étudiant l'influence des activités anthropiques sur le système terrestre, ce néologisme renvoie à la question philosophique de la place de l'être humain sur la terre et " l'anthropocène » implique dorénavant un dialogue - obligatoirement - interdisciplinaire pour traiter simultanément des enjeux sociaux, environnementaux, politiques, économiques (Choné / Hajek / Hamman 2016).

A ces titres divers, les études culturelles rencontreront nécessairement les études germaniques en France. Dans ce qui suit, on se propose d'observer de manière synthétique l'histoire de cette rencontre, en posant la présence de trois stades lors desquels s'établissent des points de rencontre plutôt que des recouvrements, et de signaler - en raccourci dans le cadre du présent article - dans quelle mesure il y a et il y a eu ces liens entre traditions repérables dans les études germaniques en France et pratiques novatrices apportées par les études culturelles.

\section{ETUDES GERMANIQUES ET ÉTUDES CULTURELLES : UNE HISTOIRE D'ABORD PARALLÈLE}

Si l'on se tourne vers l'histoire des études germaniques en France, on pourra constater d'abord qu'elles ont certes une forme d'antériorité relativement aux études culturelles. Suivant qu'on entende par "études germaniques » une forme de praxéologie ou d'herméneutique, on fera plutôt remonter les études germaniques à la première moitié du XIXe siècle (praxéologie) ou à la seconde moitié du XIXe siècle (herméneutique), en considérant soit que les premiers « germanistes » en France étaient des maîtres de langue (Espagne / Werner : 1991), soit qu'ils étaient des historiens, philosophes, comparatistes (Raulet 1994 : 380).

Toutefois, le paradoxe de cette pluridisciplinarité était plutôt qu'elle a débouché, dans un premier temps, sur des conflits de facultés, et notamment sur un tiraillement entre un recentrement philologique et une ouverture affichée à d'autres horizons pour l'enseignement et la recherche. Les disciplines représentées au sein des études germaniques françaises sont traditionnellement : la civilisation, l'histoire des idées, la linguistique, la littérature ; toutefois, la philologie littéraire qui est d'abord représentée par les ténors des études germaniques en Sorbonne s'impose longtemps comme le cœur de métier entouré des autres orientations qui vont périodiquement revendiquer une nouvelle place disciplinaire. Sans entrer ici dans des détails que l'on trouvera ailleurs, rappelons que ce fut notamment le cas des études en civilisation qui s'affirmèrent au moment de la fondation de l'Association des germanistes de l'enseignement supérieur en 1967 (Lartillot 2013 : 61-83) par la voix de grands ténors de la germanistique française tels Pierre Bertaux et Robert Minder ; d'un point de vue chronologique, ce jalon se situait dans une époque de libéralisation, héritière pour ainsi dire des mutations qui ont entouré la naissance des Cultural Studies (Lartillot 2013 : 6183). Toutefois, ce point de vue d'un nécessaire décloisonnement et renouvellement de l'enseignement et de la recherche ne s'imposera pas comme étant le seul valable et ne conduira pas à une méthodologie scientifique en propre.

De cette histoire contrastée ressortit en réalité un paradoxe qui est le miroir du paradoxe français à un niveau plus large : les études germaniques de par leur pluralité pourraient approcher des Cultural Studies, mais elles connaissent de par leur genèse des interactions avec l'histoire institutionnelle de l'enseignement et à ce titre elles restent marquées par d'autres idéaux scientifiques qui l'ont traversée. Et elles ont affirmé, entre les sciences nomothétiques et les sciences idéographiques, une tierce voie adossée à la philologie « intentionnelle » (Röseberg 2012 ; Saint-Jacques 2011 : 154). Sans revenir sur les controverses déjà anciennes et illustrées 
notamment par les critiques de Roland Barthes sur le rôle de " l'intention » dans l'interprétation littéraire, nous indiquons que l'ouvrage de Dorothee Röseberg Formation de la raison reconduit cette question même aux Lumières. Pour sa part, Denis Saint-Jacques rappelle l'action de Gustave Lanson qui a donné à la littérature ses lettres de noblesse relativement aux études scientifiques, en axant leur scientificité sur l'étude du texte. Or, si on peut considérer que la French Theory, du fait qu'elle ressortit déjà à un débat avec cette institutionnalisation de l'étude du texte - si on la fait remonter à la génération de Derrida et Foucault -, a déjà éclipsé la naissance des Cultural Studies (Neveu 2011 : 159)2 , voire qu'elle a abordé finalement des questionnements semblables par d'autres voies d'accès, on pourrait alors même affirmer que les études germaniques se situent en un lieu du « ni, ni ». C'est-à-dire qu'elles ne sont ni impliquées de plain-pied dans le débat de la French Theory, ni ne se sont affiliées dans un premier temps du moins aux Cultural Studies. Néanmoins, on ne peut pas non plus affirmer qu'elles sont entièrement indemnes de liens avec elles, ces liens dont on peut constater l'existence à l'occasion d'observations empiriques ponctuelles qui se créent à l'occasion de tournants institutionnels et d'une forte internationalisation des études et recherches, comme le confirment les nombreuses directions de thèse en cotutelle.

\section{DE L'ACCOMMODEMENT À LA TRANSITIVITÉ : DES ÉVOLUTIONS INSTITUTIONNELLES APRÈS 1990}

Le constat que nous faisons plus haut semble faire état d'une certaine frilosité des études germaniques françaises. Néanmoins, en observant l'évolution des études germaniques après 1990, ainsi que le panorama des formations et de la recherche, du moins par sélection ponctuelle, on pourrait parvenir à des observations plus nuancées. Il faudrait une étude plus approfondie pour indiquer les causes de cette mutation, dont certaines nous sont en tout cas apparentes : d'une part la chute du mur a provoqué un ébranlement des consciences dont l'un des corollaires a peut-être été le décentrement des études et la redéfinition des études germaniques ; d'autre part, les difficultés de la discipline l'ont conduite à entrer de manière plus affirmée en interaction avec d'autres disciplines en France et à l'étranger, et enfin, l'internationalisation croissante de la recherche et l'accent porté par les instances d'évaluation et de financement sur cette nécessité ont probablement infléchi et déplacé les accents portés en études germaniques tant en enseignement qu'en recherche. Sans prétendre ici dresser un tableau exhaustif de cette évolution on donnera quelques repères qui vont en ce sens.

Deux institutions illustrent cette évolution. Elles naissent de part et d'autre du Rhin, avec une vocation apparentée quoique non similaire: le CIERA (Centre Interdisciplinaire d'Etudes et

2 C'est ainsi la constatation paradoxale de l'étude d'Erik Neveu : « La ligne Paris-Londres des cultural studies : une voie à sens unique? ». de Recherche sur l'Allemagne) et le Centre Marc Bloch. Le CIERA créé le 13 décembre 2001 suivant le Journal Officiel s'inscrit dans le cadre du développement des études aréales, et promeut l'interdisciplinarité. Suivant Anne-Marie SaintGille, le CIERA travaille à penser à nouveaux frais l'interculturel ainsi que l'ancrage disciplinaire. Les activités scientifiques qui ont été menées en son sein visent en effet non seulement à une circulation des savoirs au niveau des contenus, mais aussi à dépasser les frontières disciplinaires et nationales en proposant non pas une juxtaposition des approches et des méthodes propres à chacun, mais un croisement et une réflexivité de celles-ci (Saint-Gille 2013 : 179-187).

Le Centre Marc Bloch de Berlin, centre franco-allemand et à vocation interdisciplinaire, créé en 1992, a pour caractéristique d'être une institution binationale de recherche et de formation à la recherche dans le domaine des sciences humaines et sociales. Par nécessité ces institutions illustrent une volonté d'approcher autrement les savoirs liés à l'étude des aires d'expression allemande placée, l'une sous l'égide de son père fondateur, Michael Werner, et de sa coopération avec des sociologues, l'autre sous l'égide plutôt d'Etienne François et de ses recherches sur les études mémorielles, mais transcendant naturellement les limites de ces « territoires de recherche ».

Par ailleurs, il faudrait noter le fort tropisme « interculturel » des études germaniques. Bien que les études interculturelles ne soient certes pas en elles-mêmes une institution, ce courant de la recherche s'est ancré dans le paysage des investigations menées conjointement a minima par des chercheurs allemands et français et il est apparenté à certains égards aux Cultural Studies. II n'est pas possible ici de citer toutes les études menées sous cette égide ni toutes les équipes d'accueil relevant de ce tropisme scientifique, mais on pourrait évoquer comme exemple type les interactions vives d'Hans-Jürgen Lüsebrink et de Michel Grunewald autour des interactions France-Allemagne dans le cadre de la « production de savoir sur l'autre », ou de Hans-Jürgen Lüsebrink et Michel Espagne autour de l'africanistique allemande et européenne, ainsi que d'une étude récemment lancée sur la circulation des savoirs au XVIII siècle autour des encyclopédies (qui réunit finalement divers critères de la Kulturwissenschaft) telle qu'elle peut s'établir entre autres avec des germanistes français.

Cette évolution institutionnelle ou semi-institutionnelle marque aussi un tournant sur le terrain, dont les effets sont perceptibles dans le paysage composite des " études germaniques ॥ françaises actuelles et peut-être leur conquête d'un nouveau terrain.

\subsection{A la conquête d'un nouveau « terrain »?}

Ces institutions ont donc infléchi les programmes de recherche et d'enseignement et transforment le paysage des études germaniques. On en verra des indicateurs d'évolution à trois niveaux :

- Les intitulés de master DFH

- Les intitulés de doctorat et leur contenu

- Les programmes des Équipes d'Accueil 
Bien entendu, il ne s'agit pas de voir des « études culturelles " partout. Néanmoins, on observe une porosité entre la nébuleuse des "études culturelles » et les "études germaniques » françaises, telles qu'elles s'incarnent diversement.

Le guide des études de l'Université Franco-Allemande renseigne sur les masters sélectionnés et soutenus, notamment en Sciences Humaines et Sociales (SHS). Or, dans ce secteur si l'on exclut les programmes dédiés aux sciences politiques, on observera la présence massive de masters dédiés à des phénomènes culturels ou interculturels ${ }^{3}$.

Sans être en mesure de faire un relevé exhaustif des doctorats soutenus en études germaniques ces dernières années, on pourra aussi observer une évolution de ceux-ci. Les études féministes, les études africanistes, mais aussi des objets hybrides d'études pourraient être cités et témoignent d'un déplacement du centre de gravité des études germaniques, ce déplacement est fréquemment lié à des cotutelles de thèse. Sans du tout être ici exhaustive, nous proposons quelques exemples typiques de cette évolution soit au plan thématique soit au plan méthodologique. Ce sont les thèses de doctorat, publiées, qui ont été soutenues entre autres par Anne-Laure Briatte-Peters, Lucile Dreidemy, Sonja Malzner, Pauline Landois. L'étude d'A.L. Briatte-Peters, Citoyennes sous tutelle. Le mouvement féministe "radical " dans l'Allemagne wilhelmienne (dirigée par Monique Mombert - Université de Strasbourg - et Sonia Paletschek - Universität Freiburg i. Br.), se comprend comme une contribution à l'histoire sociale de la bourgeoisie et à l'histoire politique de l'Allemagne wilhelminienne »; posant en préalable le constat que ces « radicales » entendaient exprimer leurs opinions politiques, elle démontre que, outre leurs " enjeux identitaires " de femmes et leurs enjeux " éthiques " d'individus, elles ont lutté pour leurs droits et leurs devoirs afin de prendre part à la politique et sont devenues, au bout du compte, les « championnes » de la « démocratisation du système politique ». Dans Denn ein Engel kann nicht sterben: Engelbert Dollfus 1934-2012 : eine Biographie des Posthumen (dirigée par Geneviève Humbert - Université de Strasbourg - et Oliver Rathkolb - Universität Wien), L. Dreidemy traite Engelbert Dollfuss comme objet de l'écriture politique de l'histoire et de la mémoire de l'Autriche, thématique inédite dans la germanistique française et encore peu exploitée en Autriche. S. Malzner, dans sa Dissertation "So sah ich Afrika». Die Repräsentation von Afrikanern in plurimedialen Reiseberichten europäischer Individualreisender der ersten Hälfte des 20. Jahrhunderts (dirigée par Françoise Lartillot - Université de Metz - et Hans-Jürgen Lüsebrink - Universität Saarbrücken), se place au carrefour des études aréales, littéraires et culturelles ; non seulement elle y adopte la double perspective de la romanistique et de la germanistique mais elle transfère le gain philologique à la lecture de nouveaux matériaux et aborde la relation de voyage en tant que phénomène plurimédiatique, les photographies accompagnant les textes jouant un rôle fondamental.

3 https://www.dfh-ufa.org/fr/programmes/guide-des-etudes/ (dernière consultation le 18/09/2018).
Un autre exemple est l'étude de P. Landois, Artisans autobiographes, écritures de soi dans l'artisanat allemand en mutation 1731-1815 (dirigée par Françoise Knopper - université de Toulouse - et Annette Simonis - Universität Giessen), dont l'objectif est de relier les données sociologiques, historiques, économiques, de l'artisanat avec leur manifestation dans le discours spécifique du for privé des compagnons artisans, donc en l'occurrence de non professionnels de l'écriture.

Enfin, les programmes des Équipes d'Accueil de germanistes ou comprenant des germanistes évoluent aussi dans ce sens. Là aussi, les études culturelles favorisent souvent les croisements thématiques des différentes disciplines regroupées en leur sein. Ainsi, le CREG de Toulouse (Centre de Recherche et d'Etudes Germaniques - EA 5141) sous la direction de Françoise Knopper d'abord, a choisi de décliner avec l'ensemble de l'équipe des thèmes transversaux, proches de préoccupations sociologiques. L'UMR 8131, fondée en 2001 sous l'intitulé de CRIA (Centre de Recherches Interdisciplinaires sur l'Allemagne) est devenu le Centre Georg Simmel (UMR sous tutelle du CNRS et de l'EHESS) et marque ainsi peut-être plus nettement un rapprochement à celui que l'on considère volontiers comme l'un des pères fondateurs des Cultural Studies du moins au plan épistémologique.

En guise de conclusion, un questionnement

Si les « études culturelles » empruntent volontiers le discours de la préconisation morale - et on pourrait citer comme illustration typique de cette tendance, la conclusion de Lawrence Grossberg (Grossberg 2013 : 55) :

Les Cultural Studies ne vont pas sauver le monde ou même I'Université ; au lieu de cela, elles sont un projet modeste en vue d'une pratique intellectuelle et politique flexible et radicalement contextuelle, une pratique qui essaie d'établir des connexions, ainsi que le formule Meaghan Morris, entre la politique de la culture et la politique du politique. Et en tant que telles, elles pourraient bien nous aider à nous rapprocher un peu plus de notre but, à savoir faire du monde un endroit plus juste et plus équitable pour tous

- il semblerait en tout cas légitime de s'interroger inversement sur le gain des voies empruntées.

Ainsi, la porosité constatée n'empêche pas que les germanistes français, à l'instar d'autres scientifiques, fassent montre d'une certaine vigilance en la matière. On pourrait nommer de manière exemplaire l'introduction d'Anne Chalard-Fillaudeau et Gérard Raulet qui s'interrogent sur l'« organon » de ces études culturelles tout autant que sur leur portée et validité politiques et scientifiques (Chalard-Fillaudeau / Raulet 2003 : 3-30), ou l'analyse de Marion Picker quant à l'usage échevelé de certaines métaphores qui, si elles s'émancipent, tiennent lieu de pensée tout en n'étant en fait que des analogies suggestives, ainsi de celle de la "carte mentale » et autres outils de cartographie privilégiés par les Cultural Studies (Picker 2013 : 33-46).

Toutefois, la visée sinon l'organon pourrait bien être celui d'une objectivation de la complexité sans réification, accompagnant la volonté de mettre en lumière les réifications à 
l'œuvre dans la culture et dans la société (pour reprendre une formule commentant de manière générale le positionnement de Georg Simmel lui-même (Thouard / Zimmermann 2017 : 17) en matière d'études culturelles (Chalard Fillaudeau $2015: 65)$.
Moyennant cette prudence et suivant cette inspiration, les sciences de la culture sont les hôtes bienvenus, en l'auberge (germaniste ?) d'un humanisme humboldtien perpétué, pardelà les vicissitudes de la politique universitaire.

\section{Bibliographie}

Brantlinger, Patrick (1990/2013), Crusoe's Footprints: Cultural Studies in Britain and America, London, Routledge / Hoboken, Taylor and Francis.

Briatte-Peters, Anne-Laure (2013), Citoyennes sous tutelle. Le mouvement féministe "radical " dans l'Allemagne wilhelmienne, Berne, Peter Lang.

Chalard-Fillaudeau, Anne / Raulet, Gérard (2003), « Pour une critique des "sciences de la culture »", L'Homme \& la société, 2003/3 ( $n^{\circ} 149$ ), 3-30.

Chalard-Fillaudeau, Anne (2015), Les études culturelles, SaintDenis, Presses universitaires de Vincennes.

Choné, Aurélie / Hajek, Isabelle / Hamman, Philippe (dir.) (2016), Guide des Humanités environnementales, Villeneuve d'Ascq, Presses universitaires du Septentrion.

Dreidemy, Lucile (2014), Der Dollfuß-Mythos Eine Biographie des Posthumen, Wien u.a., Böhlau.

Espagne, Michel / Werner, Michael (dir.) (1991), Le maître de langues. Les premiers enseignants d'allemand en France (18301850), Paris, MSH.

Espagne, Michel / Lüsebrink, Hans-Jürgen (dir.) (2015), Transferts de savoirs sur l'Afrique, Paris, Karthala.

Ette, Ottmar (2001), Literatur in Bewegung. Raum und Dynamik grenzüberschreitenden Schreibens in Europa und Amerika, Weilerswist, Velbrück Wissenschaft.

Grossberg, Lawrence (2003), "Le cœur des Cultural Studies ", L'Homme \& la société 2003/3 ( $n^{\circ} 149$ ), 41-55.

Landois, Pauline (2017), Artisans autobiographes. Écritures de soi dans l'artisanat allemand en mutation (1731-1817), Halle, Universitätsverlag.

Lartillot, Françoise (2013), « L'AGES de 1967 à 2009 : lieu de rencontre des germanistes français et miroir d'une discipline ", in Grunewald, Michel / Lüsebrink, Hans-Jürgen / Marcowitz, Reiner / Puschner, Uwe (éd.) : France-Allemagne au XXe siècle - La production de savoir sur l'Autre, vol. 3, Berne, Peter Lang, 61-83.

Malzner, Sonja (2013), ,So sah ich Afrika: Die Repräsentation von Afrikanern in plurimdialen Reiseberichte der ersten Hälfte des 20. Jahrhunderts, Würzburg, Königshausen \& Neumann.
Marcowitz, Reiner / Puschner, Uwe (éd.) (2013), France-Allemagne au XXe siècle - La production de savoir sur l'Autre, vol. 3, Berne, Peter Lang, 61-83.

Mattelart, Armand / Neveu, Erik (2010), Introduction aux cultural studies, Paris, La Découverte.

Michaud, Yves (2006), Humain, inhumain, trop humain, réflexions philosophiques sur les biotechnologies, la vie et la conservation de soi à partir de l'œuvre de Peter Sloterdijk, Paris, Climats.

Musner, Lutz (2004), Kultur als Textur des Sozialen. Essays zum Stand der Kulturwissenschaften, Wien, Löcker.

Neveu, Erik [2002] (2011), « La ligne Paris-Londres des cultural studies : une voie à sens unique ? ", in Cohen, Evelyne / Goetschel, Pascale / Martin, Laurent / Ory, Pascal (dir.) : Dix ans d'histoire culturelle, Villeurbanne, Presses de l'enssib, 159-173.

Picker, Marion (2013), « Le 'Mapping' et ses limites. Réflexions sur une métaphore méthodologique dans les sciences de la culture "; in Lartillot, Françoise / Pfeil, Ulrich (éd.), Constructions de l'espace dans les cultures d'expression allemande. Peter Lang, Bern, 33-46.

Raulet, Gérard (1994), "L'histoire des idées. Situation et fonctions » in : Werner, Michael / Espagne, Michel (dir.), Les Etudes germaniques en France, Paris, CNRS Ed., 379-395.

Röseberg, Dorothee (2012), Formation de la raison. Zur Geschichte französischer Wissenskultur aus interkultureller Perspektive (18. 21. Jh.), hrsg. von Stiebritz, Anne, Jena, Paideia.

Saint-Gille, Anne-Marie (2013), « Pour l'émergence d'une nouvelle génération de chercheurs sur l'Allemagne : le projet et les activités du CIERA ", in Grunewald, Michel / Lüsebrink, HansJürgen / Marcowitz, Reiner / Puschner, Uwe (éd.) (2013), FranceAllemagne au XXe siècle - La production de savoir sur l'Autre, vol. 3, Berne, Peter Lang, 179-187.

Saint-Jacques, Denis (2011), "La contrebande disciplinaire », in Cohen, Evelyne / Goetschel, Pascale / Martin, Laurent / Ory, Pascal (dir.) : Dix ans d'histoire culturelle, Villeurbanne, Presses de l'enssib, 153-158.

Thouard, Denis / Zimmermann, Bénédicte (2017), "Considérations simmeliennes ", in Thouard / Zimmermann, Simmel, le parti pris du tiers, CNRS éditions, 2017. 